\title{
Triple-point wetting of molecular hydrogen isotopes
}

\author{
Masoud Sohaili ${ }^{1}$, Jürgen Klier and Paul Leiderer \\ Department of Physics, University of Konstanz, D-78457 Konstanz, Germany \\ E-mail: masoud.sohaili@uni-konstanz.de
}

Received 24 November 2004

Published 18 February 2005

\begin{abstract}
Triple-point wetting is a well-known phenomenon of simple adsorbates on solid substrates, which involves, in the liquid phase above the triple-point temperature, $T_{3}$, complete wetting with the formation of arbitrary thick films being observed, whereas below $T_{3}$ only a few monolayers of the solid phase are adsorbed at saturated vapour pressure. This effect is usually ascribed to the substrate-induced strain in the solid film, which occurs due to the lattice mismatch and the strong van der Waals pressure in the first few monolayers. Molecular hydrogen is a suitable system in which to investigate this phenomenon, in particular by tailoring the adsorbate-substrate interaction by means of thin preplating layers of other adsorbates, and by introducing disorder into the system by using not only the pure systems $\mathrm{H}_{2}$ and $\mathrm{D}_{2}$, but also mixtures thereof. The experiments show that triple-point wetting is a rather dominant effect which, in contrast to expectations, persists even if the system parameters are widely varied. This indicates that the present understanding of this effect is incomplete. We present an investigation of the influence of the roughness of the substrate which is expected to be responsible for the dewetting of the solid phase.
\end{abstract}

\section{Introduction}

Wetting and dewetting of physisorbed films have been studied extensively both theoretically [1-5] and experimentally [8-25]. A number of reviews on this subject, like those by Dietrich [6] and Schick [7], exist. There are two ways in which multilayer films can grow on an attractive substrate. In the first, the thickness of the film increases with increasing pressure, and diverges as the vapour pressure approaches the saturated value (complete wetting). In the second, the thickness of the film remains finite at bulk coexistence (incomplete-partial wetting). For a given adsorbate and substrate combination a transition may occur from incomplete to complete wetting as a function of temperature [2].

1 Author to whom any correspondence should be addressed.

Konstanzer Online-Publikations-System (KOPS)

URL: http://www.ub.uni-konstanz.de/kops/volltexte/2007/2731/

URN: http://nbn-resolving.de/urn:nbn:de:bsz:352-opus-27316 
Triple-point wetting is described as a transition from an incomplete wetting solid film to a complete wetting liquid film at its triple point, $T_{3}$ [14]. In the early theories lattice mismatch between the film and the substrate was considered to be responsible for the finite thickness of the solid film $[4,5]$.

The majority of measurements have been devoted to adsorption isotherms of rare gases on graphite [8-10], $\mathrm{Ag}$ [11], $\mathrm{MgO}$ [12], $\mathrm{CO}_{2}$ [13], and $\mathrm{Au}[14,15]$, which were mostly in the thickness range of one to ten monolayers. Multilayer $\mathrm{H}_{2}$ films have been studied on graphite substrates via volumetric adsorption isotherms [16] and ellipsometry [17] or on $\mathrm{Ag}$ via a quartz crystal microbalance [18]. Intensive investigations of $\mathrm{H}_{2}$ on $\mathrm{Au}$ and preplated $\mathrm{Au}$ substrates have been the subject of a series of experiments [19-21] in which the surface plasmon spectroscopy technique was used. Films of $\mathrm{H}_{2}-\mathrm{D}_{2}$ mixtures [22, 23] and thick quench-condensed films [24-27] have been investigated via these methods. A few measurements $[28,29]$ have also been performed on the surface of $\mathrm{Si}$. There are hints that strain in the solid films, induced by surface roughness, is important for $T_{3}$ dewetting, in addition to the lattice mismatch effects mentioned above.

Thick solid films of $\mathrm{H}_{2}$ isotopes have subtle fundamental applications and it is therefore also important from this practical point of view to understand the incomplete wetting of these films below $T_{3}$. Solid $\mathrm{H}_{2}$ films can be used as a substrate [30-32] to hold electrons at a distance above them. Preparation of thick solid $\mathrm{T}_{2}$ films plays a decisive role in experiments designed to measure the neutrino rest mass [33-35] and solid $\mathrm{D}_{2}$ films in nuclear fusion [36, 37]. Moreover, $\mathrm{H}_{2}$ films, which due to their light molecular mass have a quantum nature, offer another system for studying relationships which may exist between the quantum nature of a system and its wetting properties. For example, $\mathrm{H}_{2}$ superfluidity on relatively thick solid layers of $\mathrm{H}_{2}$ has been predicted $[38,39]$.

The outline of this paper is as follows. In section 2 we briefly give some theoretical background and recent advances which are related to this work. The experimental methods used for the measurements are explained in section 3. Achievements and results are presented and discussed in section 4 .

\section{Theoretical background}

In contrast to chemisorption, where chemical bounds are formed upon adsorption, physisorption involves formation of one or more layers of an adsorbate on a surface attracted by the substrate potential. Since typical potential depths range from a few kelvins to a few $100 \mathrm{~K}$ [40], depending on the system, physisorption can be initiated by sufficiently lowering the temperature such that the thermal excitations become small compared to the potential depth of the substrate. In general, the interaction potential between two atoms or molecules at a distance from each other is described by the Lennard-Jones (LJ) potential [41].

In the case of an atom (or molecule) a distance $z$ away from a surface the proper interaction potential, $\phi(z)$, can be obtained by summing over all pairwise LJ-based interactions of the single adsorbate with the substrate atoms filling the half-space. The calculation [42] gives

$$
\phi(z)=\frac{4 C_{3}^{3}}{27 D^{2} z^{9}}-\frac{C_{3}}{z^{3}},
$$

where $C_{3}$ and $D$ are the van der Waals (vdW) coefficient and the potential depth, respectively. At large distances the attractive part of this potential becomes dominant. This term has a $z^{-3}$ dependence, and so a weak long range interaction. This potential explains the wetting of liquid adsorbates on solid substrates correctly.

When studying the wetting of solid adsorbates on solid substrates the effect of the substrate on wetting becomes more crucial, since in this case the structural mismatch between the 


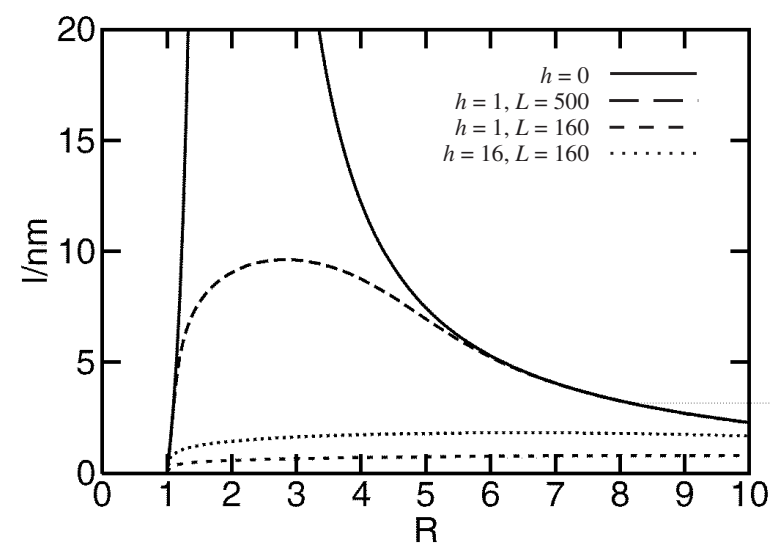

Figure 1. Comparison between the result of the former theory [5] and those of [43], for four different scales of roughness. The thickness of the solid film, $l$, increases as roughness vanishes. The parameters $h$ and $L$ are typical scales for the substrate roughness as explained in figure $2 ; R$ is the relative substrate strength.

substrate and the adsorbate causes so-called strain-induced forces in the adsorbed film. In this respect, the theory given by Gittes and Schick (GS) [5] has initially addressed the case of solid noble gases on graphite. This has recently been elaborated on and adapted for other practical substrates by Esztermann et al [43]. Since these theories are of crucial importance to the measurements reported in this paper, we briefly point out their principles and predictions. The equilibrium thickness is calculated by minimizing the total excess free energy $\Sigma\left(l_{\mathrm{s}}, l_{1}\right)$ with respect to $l_{\mathrm{s}}$ and $l_{1}$, the solid and liquid thicknesses of the adsorbate, respectively. By definition, $\Sigma=\Omega / A$, where $\Omega$ is the excess grand canonical free energy relative to a non-wetting situation and $A$ is the surface area.

The total excess free energy may be split into three different terms:

$$
\Sigma_{\text {total }}=\Sigma_{\text {thrm }}+\Sigma_{\mathrm{GS}}+\Sigma_{\mathrm{EL}}
$$

where $\Sigma_{\text {thrm }}$ results from thermodynamics. $\Sigma_{\mathrm{GS}}$, calculated by GS, is caused by the lateral induced strain in the solid adsorbate. $\Sigma_{\mathrm{EL}}$, considered as an additional term by [43], accounts for the free energy cost, caused by substrate roughness.

In figure 1, the results from both theories are shown. Plotted is the equilibrium thickness of the adsorbate versus the relative substrate strength $R$. The solid curves are the result of GS, i.e., the first two terms in equation (2). The dashed curves are the results from [43], taking a finite substrate roughness into account.

The experimental results display serious deviations from the prediction of GS. For example, as will be shown in section 4 , a solid $\mathrm{H}_{2}$ film always dewets a gold substrate below its $T_{3}$, and the thickness of the $\mathrm{H}_{2}$ film becomes of the order of a few molecular layers. According to the GS theory a considerable film thickness of around $100 \AA$ (essentially complete wetting) should be achievable for $\mathrm{H}_{2}$ on $\mathrm{Au}$ (at $R \approx 4.5$ ); see figure 1 . One possible reason for the discrepancy could be that the GS theory ignores substrate roughness which is a general property of real surfaces. This, as a result, causes an additional bending of the adsorbed solid film, considered by the new theory in [43]. Depending on the finite roughness of the substrate, the wetting of a solid adsorbate is suppressed, and the solid adsorbed film becomes thinner as the roughness scale increases. For typical substrates, the surface modulation varies on a characteristic lateral length scale $L$ which is much larger than the vertical valley to peak distance $h$, i.e., $L \gg h$ (see figure 2 ). 


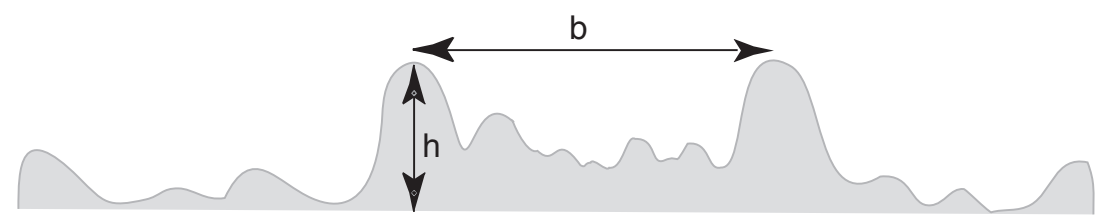

Figure 2. A schematic drawing of a roughness profile of a typical substrate. Here $h$ is the average height of the hills and $L$ the horizontal correlation length. The ratio of $h / L$ can be interpreted as a crude measure of the roughness (see [43] for the exact definition). The drawing is not to scale.

\section{Experimental procedure}

In our measurements, we used two well-known techniques for determining the film thickness, namely surface plasmon spectroscopy (SPS) and ellipsometry. SPS has been applied for measuring film thicknesses on the surface of Au [23], while ellipsometry has been used for measurements on the surface of Si [28]. The substrate was mounted vertically inside a copper cell. The adsorbate was condensed into the cell through two small capillaries which were fed into the cell from the top. The capillaries, which also served as evacuation lines of the cell, were then blocked after completion of the adsorption. The optical access to the surface was through optical windows symmetrically placed in a fourfold geometry. The experimental cell was mounted into a ${ }^{4} \mathrm{He}$ cryostat.

\subsection{Surface plasmon spectroscopy}

Surface plasmon spectroscopy is done by using P-polarized laser light on Au substrates. The light beam is resonantly coupled into surface plasmons at a certain incidence angle $[44,45]$ and focused on a photodiode placed outside of the cryostat. When a hydrogen film is adsorbed this causes a resonance detuning. Measuring the shift of the resonance angle allows us to determine the average thickness of the film adsorbed on the surface. Figure 3 illustrates schematically the SPS set-up. Using this method, thicknesses of less than a monolayer up to a few hundred nanometres can be resolved.

\subsection{Ellipsometry and laser annealing}

For measurements on $\mathrm{Si}$, a standard null-ellipsometry set-up was built up. This worked on the basis of the minimization of the reflected intensity upon reflection from the surface [46].

In figure 4 a schematic drawing of the ellipsometry set-up is shown. The ellipsometer is in a usual PCSA configuration, where P, C, S, and A stand for polarizer, quarter-wave plate, sample, and analyser (see also the figure caption). In a conventional null ellipsometer, the minimum (null) intensity, detected by a photodetector, can be obtained by properly setting A and P. Then, during adsorption the continuous rotational adjustment of $\mathrm{A}$ and $\mathrm{P}$ allows one to stay always at the minimum. By using this configuration one can extract the angles $\Psi$ and $\Delta$ from the rotation $\mathrm{A}$ and $\mathrm{P}$ angle values [47], from which the thickness of the films can be determined. We adopt the two linear approximation relations from Archer [48] for calculating the coverage. We note that this approximation holds as long as the film thicknesses do not exceed $100 \AA$. In experiments presented here, the overall thickness is always well below this value.

In addition to the ellipsometer set-up, another cw laser was used with which the local desorption-adsorption of the film could be manipulated (see figure 4). The reasons for this are discussed in section 4. Since studying the effect of surface roughness on the wetting behaviour 


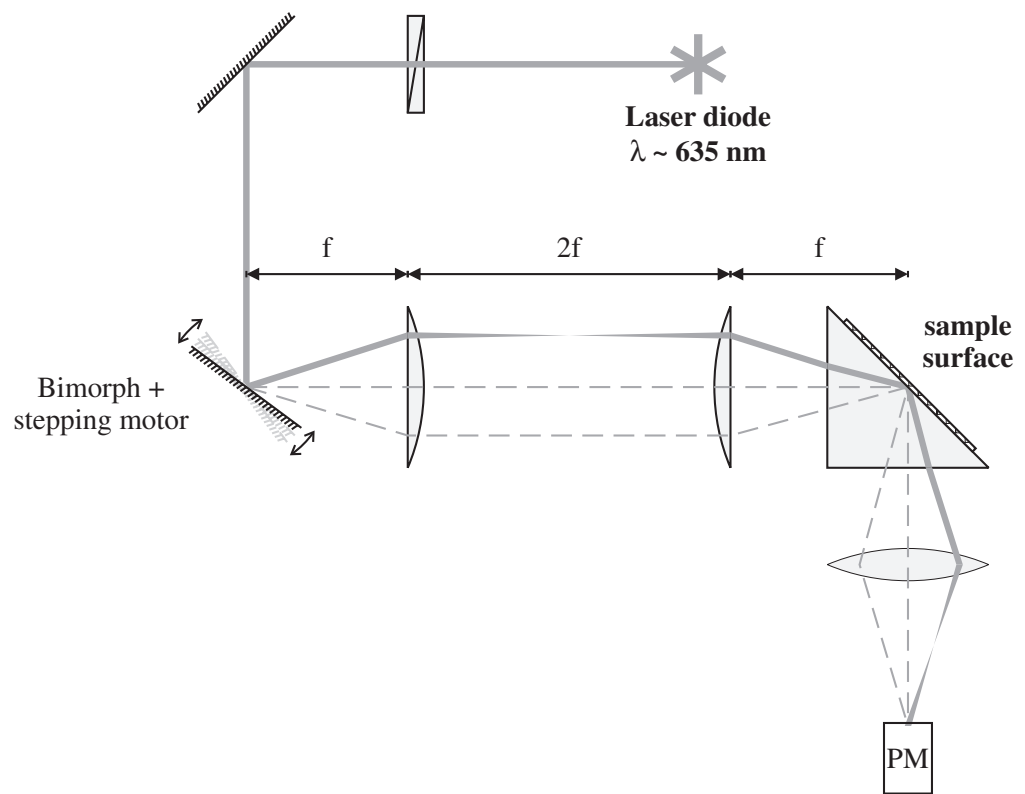

Figure 3. The main optical components of the surface plasmon spectroscopy set-up. The Au substrate, evaporated on the base of the prism, is placed inside an optical cryostat, cooled with ${ }^{4} \mathrm{He}$.

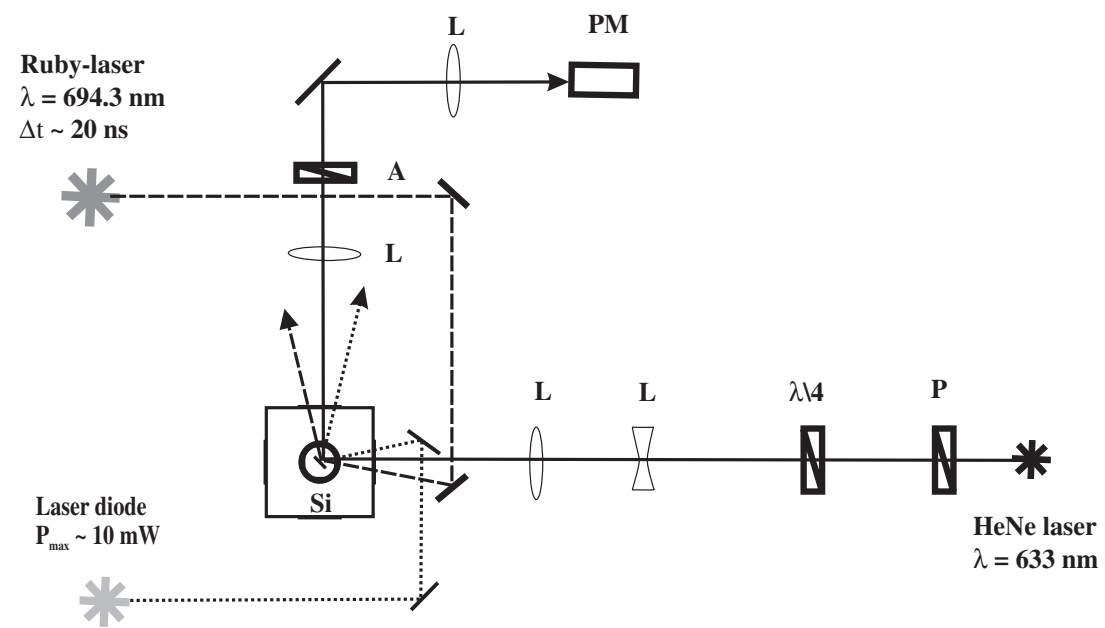

Figure 4. The set-up for the ellipsometry and the laser annealing. The main components of the ellipsometer are drawn with a stronger contrast. The system works in a PCSA configuration, where $\mathrm{P}, \mathrm{C}, \mathrm{S}$, and A stand for polarizer, quarter-wave plate $(\lambda / 4)$, substrate $(\mathrm{Si})$, and analyser. The symbols PD, PM, and L are for the photodiode, the photomultiplier, and the lens. See the text for detailed explanations of the function of each component.

of adsorbates has been the goal of this work, in situ cleaning and annealing of Si have also been done and the details are discussed in section 4.2.4. The annealing set-up is shown in figure 4 , together with the ellipsometry set-up (see $[49,50]$ for detailed information on laser annealing). 


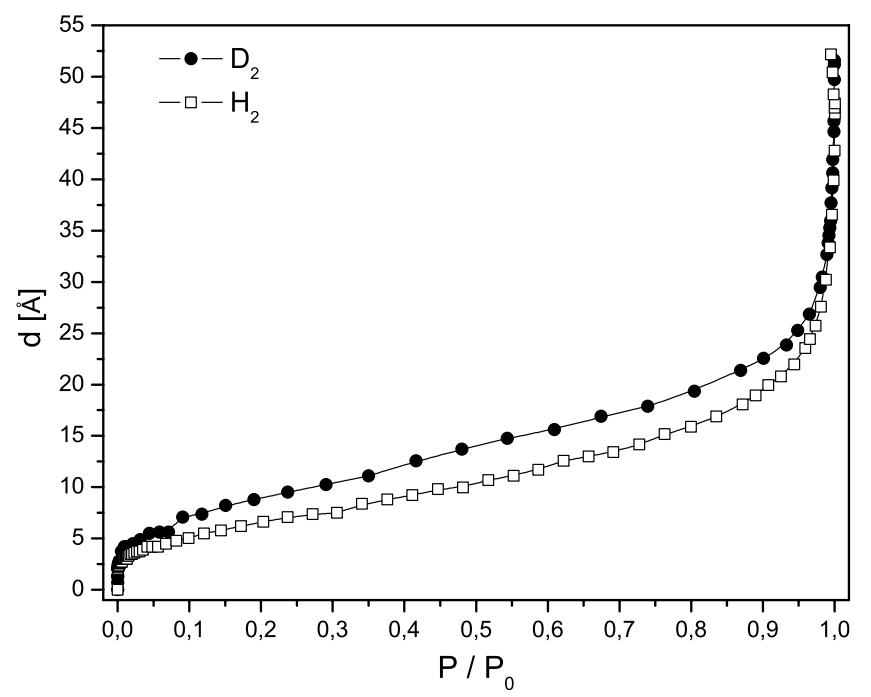

Figure 5. Adsorption isotherms of $\mathrm{H}_{2}$ and $\mathrm{D}_{2}$ at temperatures above the $T_{3}$ of $\mathrm{H}_{2}$ and $\mathrm{D}_{2}$. The thickness diverges as the saturated vapour pressure, $P_{0}$, is approached.

\section{Results and discussion}

In all the experimental runs presented here, first an adsorption isotherm was taken at a temperature well above the triple temperature of the respective adsorbate, as in earlier experiments [21-23, 28]. A sufficient amount of gas was condensed into the system that gas-liquid coexistence was reached, and bulk liquid formed at the bottom of the sample cell. (We should note that the films investigated were not exactly at coexistence, because the position of the optical measurement was located a few $\mathrm{mm}$ above the bulk surface, and furthermore the temperature of the film was slightly higher than the cell temperature due to unavoidable heating by the probe laser beam. This effect is not relevant, however, for relatively thin films below about $50 \AA$, which are the focus of this paper.) After taking the adsorption isotherm the cell was slowly cooled to temperatures well below $T_{3}$, with the amount of material in the cell being kept fixed, so the adsorbed film always had a thickness close to the equilibrium value at coexistence.

\subsection{Adsorption isotherms above $T_{3}$}

The results presented in this section are for the adsorption of $\mathrm{H}_{2}$ and $\mathrm{D}_{2}$ on the surface of a $45 \mathrm{~nm}$ thick Au layer evaporated onto a glass substrate (see figure 3). The data were taken using the SPS technique. Figure 5 shows the film thickness plotted as a function of the reduced pressure $P / P_{0}$, where $P_{0}$ is the saturated vapour pressure at the corresponding temperature. As the pressure is increased, the thickness first grows rapidly, then reaches an intermediate region of moderate growth, until eventually, close to $P_{0}$, it starts to diverge. This behaviour is qualitatively accounted for by the Frenkel-Halsey-Hill (FHH) theory [5153], which predicts a logarithmic relationship between the film thickness $d$ and the ambient pressure, given by $\alpha d^{-3}=k_{\mathrm{B}} T \ln \left(P_{0} / P\right)$. Here, $\alpha$ and $k_{\mathrm{B}}$ are the Hamaker and Boltzmann constants, respectively. As shown by Vorberg et al [54], deviations in the adsorption isotherm from the FHH prediction which are observed in the intermediate region can be explained by a contribution of thermally excited capillary waves [55]. The slight difference in the film 


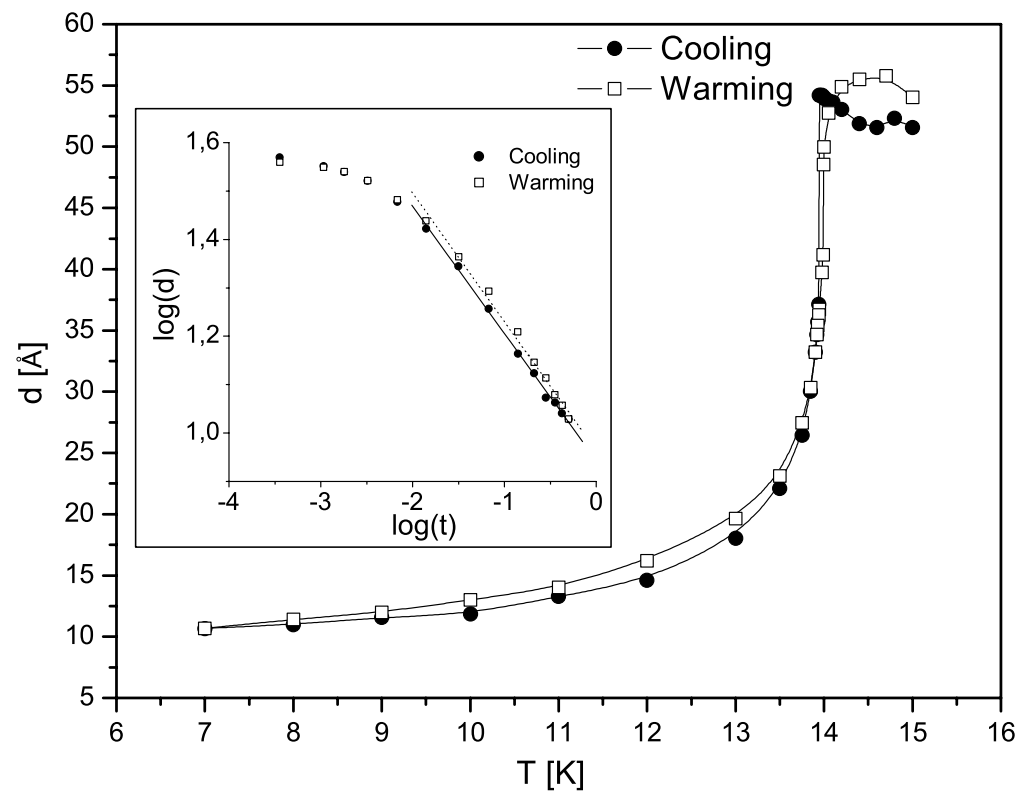

Figure 6. The temperature dependence of the film thickness of $\mathrm{H}_{2}$ adsorbed on Au. In the inset, the same data are plotted on a logarithmic scale. Here $t$ is the reduced temperature, defined as $t=\left(T_{3}-T\right) / T_{3}$.

thickness of $\mathrm{H}_{2}$ and $\mathrm{D}_{2}$ in the intermediate region of figure 5 might also be due to thermal excitations, but more quantitative studies are necessary to draw final conclusions.

\subsection{Wetting behaviour below $T_{3}$}

4.2.1. $\mathrm{H}_{2}$ and $\mathrm{D}_{2}$ on $\mathrm{Au}$. The main goal of this work was to investigate the adsorbed films close to coexistence in the temperature range below $T_{3}$, where the bulk material is solid. Typical heating/cooling cycles for $\mathrm{H}_{2}$ and $\mathrm{D}_{2}$ on a gold substrate are shown in figures 6 and 7 , respectively. The rate of the temperature change was controlled as typically $20 \mathrm{mK} \mathrm{min}{ }^{-1}$, which ensured conditions close to thermodynamic equilibrium. The behaviours observed for the two adsorbate materials are quite similar: whereas above $T_{3}$ the adsorbed film is rather thick with an essentially constant value of $d$, the thickness drops steeply upon cooling below $T_{3}$, and at low $T$ eventually levels off at a value around $10 \AA$, corresponding to about three molecular monolayers. Only a slight hysteresis between cooling and warming is seen, which confirms that the data were taken close to equilibrium. This triple-point wetting behaviour has been observed to be universal for almost all van der Waals adsorbates, in particular the noble gases [11-13]. It can be explained by assuming that only a few layers of the film are solid (which determine the thickness at $T \ll T_{3}$ ). The increase in thickness close to the triple point is ascribed to a liquid layer on top of the solid, which grows quickly as the liquid-gas coexistence vapour pressure is approached at $T_{3}$. On this basis, one expects the temperature dependence of the film thickness close to $T_{3}$ to follow a relation $d(T) \propto\left[\left(T_{3}-T\right) / T_{3}\right]^{-\beta}$, with $\beta=1 / 3$ [20]. The insets in figures 6 and 7, where the data are plotted on a logarithmic scale, show that the data do indeed follow a power law over a considerable temperature range, and that the exponent is in reasonable agreement with the prediction: in the case of $\mathrm{D}_{2}$ the fit yields $\beta=-0.30 \pm 0.003$ and $0.29 \pm 0.003$ for warming and cooling, respectively, and in the case of $\mathrm{H}_{2}$ the corresponding values are $\beta=-0.26 \pm 0.005$ and $-0.27 \pm 0.008$. 


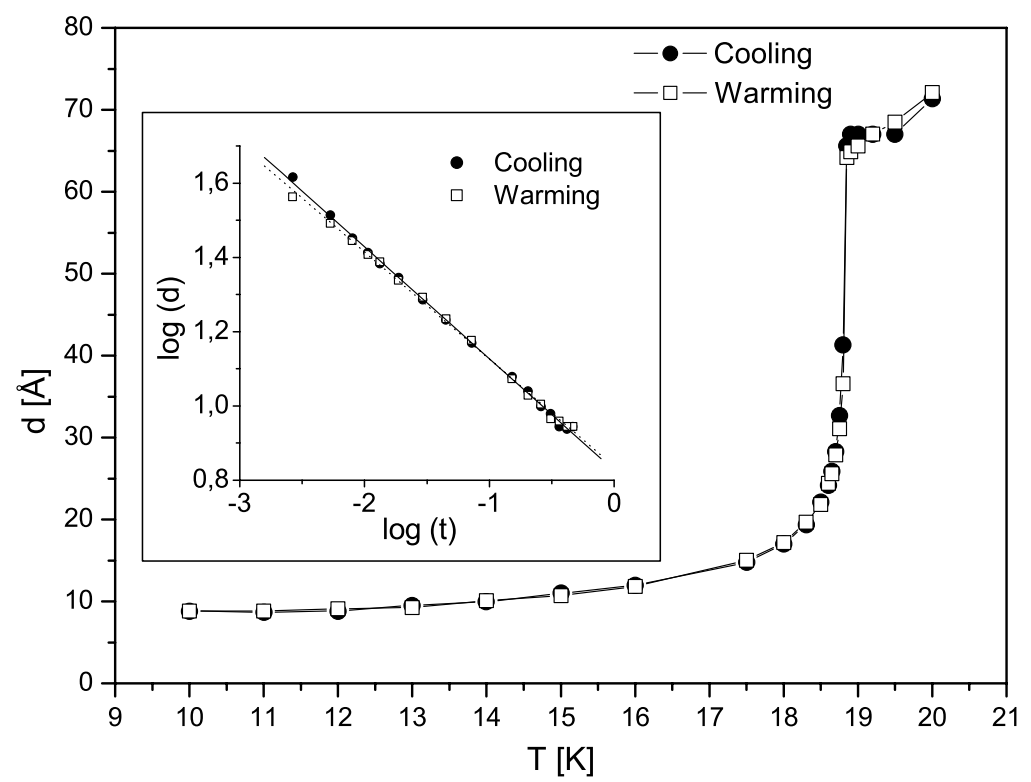

Figure 7. Wetting curves of $\mathrm{D}_{2}$ on $\mathrm{Au}$ are shown. A similar behaviour has been observed for other kinds of adsorbates. In the inset, the same curves are plotted on a logarithmic scale.

At this point the comparison between the measured solid thickness at $T \ll T_{3}$ and the prediction of theory appears adequate: for $\mathrm{H}_{2}$ on $\mathrm{Au}$ the value of the reduced substrate strength is $R=4.5$ [43]. As seen from figure 1, for this value of $R$ the solid film should be thicker than $100 \AA$, corresponding to 30 monolayers, if we assume a smooth substrate as in the GS theory [5]. From the fact that experimentally only a solid layer of about three monolayers is observed, we conclude that the substrate roughness plays an essential role here [43] (see the dotted curve in figure 1). Thus evaporated gold films, although optically smooth, appear rough on the atomic scale which is relevant for the wetting behaviour. This aspect is further investigated below for Si substrates, which are considerably smoother than our gold films (see section 4.2.3).

4.2.2. Preplating and mixtures. 'Preplating' means depositing a few monolayers of another adsorbate in advance, before the adsorption of the main adsorbate under investigation. By doing this, the interaction between the substrate and the adsorbate can be tailored, and by choosing a proper preplating material an effective substrate strength $R_{\text {eff }}$ which is smaller than the $R$ without preplating may be achieved. This could lead to wetting of the solid, and is indeed another aspect of the GS theory, which predicts a different solid thickness depending on the kind of preplating adsorbate [5].

For $\mathrm{H}_{2}$ on $\mathrm{Au}$, preplatings with $\mathrm{Ar}, \mathrm{Ne}$, and $\mathrm{CH}_{4}$ have been done already, some time ago [21]. Similar experiments followed for $\mathrm{D}_{2}$ on $\mathrm{Al}$ and graphite (HOPG) with $\mathrm{Ar}, \mathrm{Ne}, \mathrm{CH}_{4}$, and $\mathrm{C}_{2} \mathrm{H}_{6}$ [26]. Although in some cases a slight increase of the solid thickness was achieved, the overall effect of dewetting by the solid film continued to exist [21]. We have also studied the effect of $\mathrm{Ar}$ and $\mathrm{CH}_{4}$ preplated $\mathrm{Au}$ on the $\mathrm{H}_{2}$ wetting. Our results agree with previous experiments. In addition, we used $\mathrm{D}_{2}$ as a preplating material, and investigated the $\mathrm{H}_{2}-\mathrm{D}_{2}$ mixed systems. This was done by taking adsorption isotherms of $\mathrm{D}_{2}$ above its $T_{3}$. Then, the temperature was lowered and stabilized at some temperature near, but still well above, 


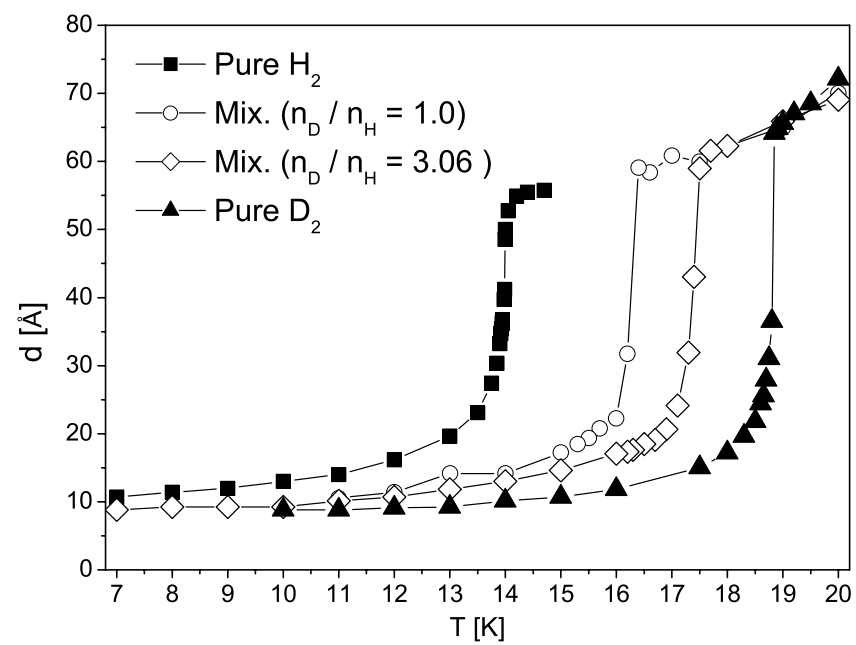

Figure 8. Wetting curves of pure and mixed systems of $\mathrm{H}_{2}$ and $\mathrm{D}_{2}$. The data demonstrate the existence of an effective triple point, $T_{3}^{(\text {eff })}$, which depends on the species concentration of the $\mathrm{H}_{2}-\mathrm{D}_{2}$ mixture. The value of $T_{3}^{(\text {eff })}$ always falls between $T_{3}^{\left(\mathrm{H}_{2}\right)}$ and $T_{3}^{\left(\mathrm{D}_{2}\right)}$.

the $T_{3}$ of $\mathrm{H}_{2}$. At this point a thin solid layer of $\mathrm{D}_{2}$ has formed on the surface of Au. The adsorption of $\mathrm{H}_{2}$ on top of the existing $\mathrm{D}_{2}$ film until saturation was reached followed before temperature scans below the $T_{3}$ of $\mathrm{H}_{2}$ were carried out. The temperature was then increased and stabilized at about $30 \mathrm{~K}$, a temperature far above $T_{3}$ of $\mathrm{D}_{2} . \mathrm{A} \mathrm{H}_{2}-\mathrm{D}_{2}$ mixture with certain species concentration was prepared and the whole system was kept at this temperature for hours. This was done to obtain a homogeneous mixture. The speed at which the temperature was changed was again about $20 \mathrm{mK} \mathrm{min}{ }^{-1}$.

The results of these experiments are presented in figure 8 (see [22, 23] for detailed discussions). It was found that an effective triple point $T_{3}^{\text {(eff) }}$ can be defined for a mixed system which falls always between the $T_{3}$ of $\mathrm{H}_{2}$ and $\mathrm{D}_{2}$, depending on the concentration of the species. Additionally, no significant change in the thickness of the solid layer is observed.

As a consequence of these preplating and mixture experiments we conclude that the dewetting below $T_{3}$ is a rather robust phenomenon which for the substrates discussed so far hardly depends on subtleties such as details of the interaction strength. This suggests that a more general aspect dominates here. We show in the next section that the key to the problem appears to be the substrate roughness, as already indicated above.

4.2.3. Wetting on $\mathrm{Si}$. We now discuss the results for the wetting of Si substrates, which are very smooth and can, in addition, conveniently be cleaned by laser annealing. The typical rms roughness of an Au substrate is measured to be about $1.3 \mathrm{~nm}$; for commercial Si wafer surfaces it is $\sim 0.15 \mathrm{~nm}$ [28], one order of magnitude smoother. The van der Waals interactions for $\mathrm{H}_{2}$ on $\mathrm{Au}$ and $\mathrm{Si}$ are nearly the same [40, 56-58]. Using the calculations of Vidali and Cole [56] we get 8739 and $6276 \mathrm{~K}^{3}$ for $\mathrm{H}_{2} / \mathrm{Au}$ and $\mathrm{H}_{2} / \mathrm{Si}$, respectively, with estimated uncertainties of about $20 \%$ arising from the applied approximations. Considering the fact that the film thickness is proportional to the cubic root of the vdW coefficient, Si and Au should essentially behave similarly with respect to substrate strength. It is therefore likely that-should differences in the wetting behaviour of our Au and Si surfaces be observed-they can be ascribed to the different surface topography. 


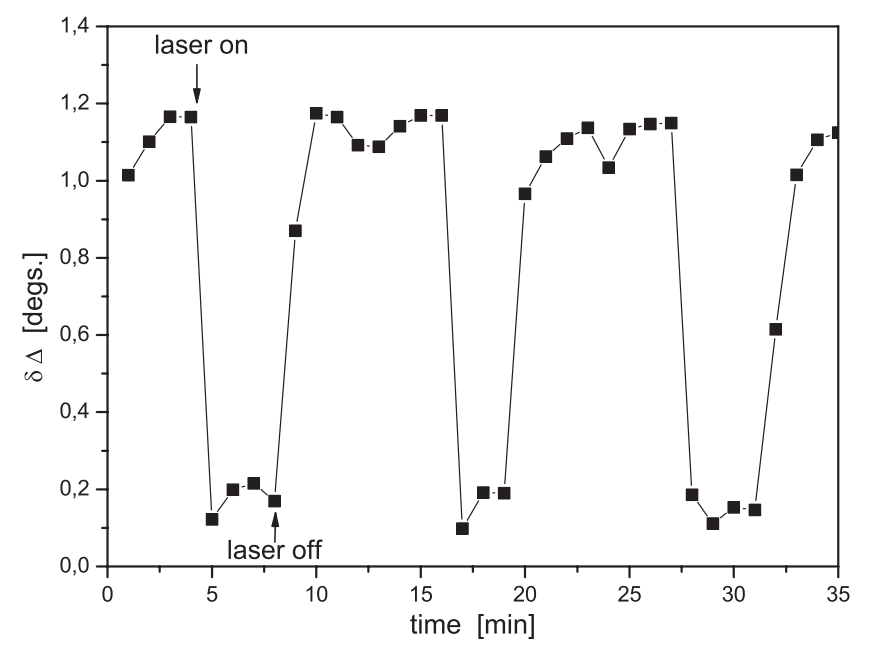

Figure 9. A real time snapshot of the change of the ellipsometer angle $\delta \Delta$ (and hence the film thickness) of a saturated liquid $\mathrm{H}_{2}$ film on $\mathrm{Si}$ upon laser heating is shown. The temperature is about $14 \mathrm{~K}$, slightly above $T_{3}$ for $\mathrm{H}_{2}$. The thickness decreases (as $\delta \Delta$ decreases) as the heating laser is switched on, and comes back to the original value before heating when the heating laser is turned off.

The measurements on $\mathrm{Si}$ were done by means of ellipsometry as described in section 3.2. The ellipsometer is very sensitive to polarization effects, such as strain-induced birefringence in the quartz windows, and, as a result, the position of the minimum may shift upon temperature ramping. In order to reduce such effects due to drift, we applied in this case a differential method: in addition to the ellipsometer probe laser (power $100 \mu \mathrm{W}$ ) a heating laser $(10 \mathrm{~mW}$ ) was focused onto the probe spot, which was periodically switched on and off at a frequency of $3 \mathrm{mHz}$ (see figure 9). In the 'on' state the intensity of the heating laser was sufficient to desorb the major part of the adsorbed film (apart from three monolayers which appeared to be more strongly bound). In the 'off' state the film quickly recovered to its initial thickness without a heating laser, as shown in figure 9. The difference between 'on' and 'off' thus provides information about the film thickness (apart from the remaining strongly bound layer) free of drift effects. The data in figures 10-12 were obtained with this method.

Figure 10 shows results for $\mathrm{H}_{2}$ on $\mathrm{Si}$ determined in this way. The thickness saturates below $13 \mathrm{~K}$ at a value of 0.32 in units normalized to the thickness above $T_{3}$ (which is $55 \pm 5 \AA$ ), corresponding to $17.5 \AA$. Compared to the value on rough $\mathrm{Au}(d \approx 10 \AA)$ the thickness is thus significantly increased. This effect is also illustrated in figure 11, which shows the film thickness on $\mathrm{Si}$ on a logarithmic scale, like in the inset of figure 6. Again a power law is observed with a value of $\beta=0.31 \pm 0.02$ in this case, but in contrast to the case for figure 6 the data display a clear deviation from this law for $\log (t)>-1.60$, where the thickness starts to saturate. We interpret this saturation at low temperatures as evidence for the solid $\mathrm{H}_{2}$ forming a distinctly thicker film on the smooth Si substrate.

Similar measurements were done for $\mathrm{D}_{2}$ on $\mathrm{Si}$, and the data, shown in figure 12 , corroborate the results for $\mathrm{H}_{2}$.

4.2.4. Laser cleaning and annealing. Here we discuss our first results obtained from measurements on in situ cleaned and annealed Si substrates. The idea of the laser cleaning is to remove unwanted contaminations (e.g. water) from the Si surface, which adsorb while mounting the substrate into the cell at room temperature and ambient atmosphere or during 


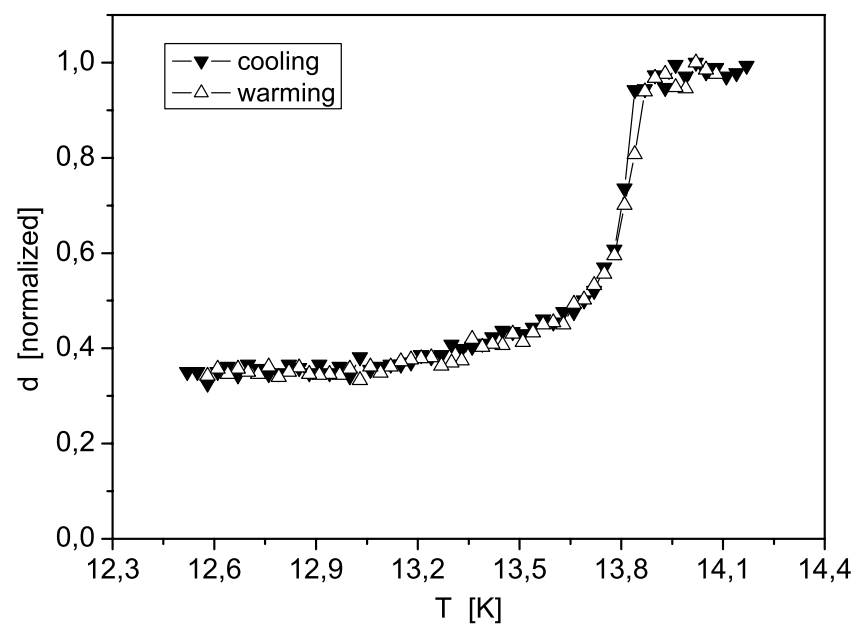

Figure 10. Shown is the wetting behaviour of $\mathrm{H}_{2}$ on $\mathrm{Si} . \quad d$ is normalized to the thickness in saturation above $T_{3}$ of $\mathrm{H}_{2}$.

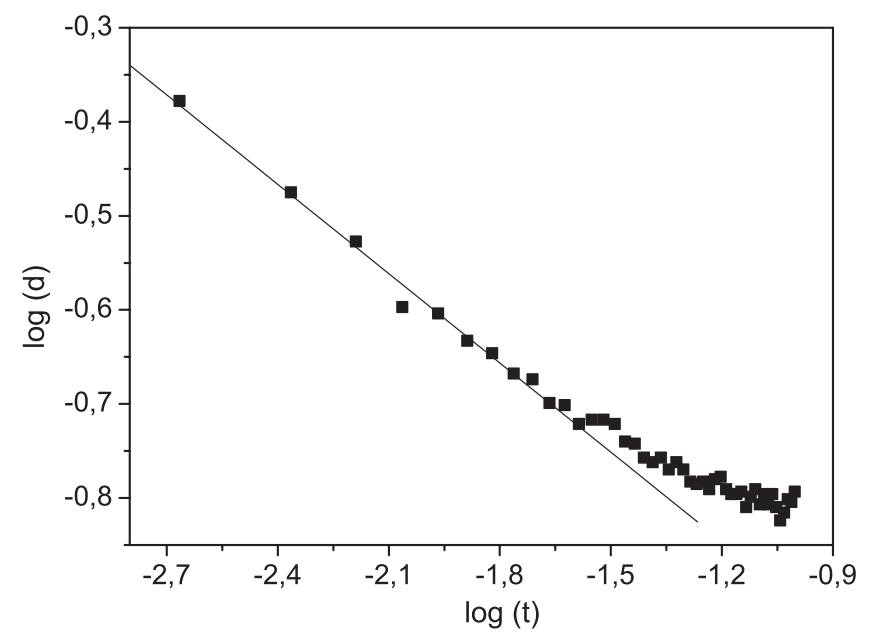

Figure 11. Illustrated is the warming curve of figure 10 plotted on a logarithmic scale. The slope of the straight line is 0.31 .

cooling down the cell (we do not have UHV conditions). For the cleaning procedure, we focus the light of a powerful laser diode $(200 \mathrm{~mW})$ on the same area of the substrate where the ellipsometry beam hits the surface. This is done at the beginning of the experiment, and the laser light is continuously left on while cooling the cell from room temperature down to the working temperature. In this way we can keep the temperature of the substrate about $30 \mathrm{~K}$ above the ambient temperature in the cell. The colder cell walls thus act as a trap for any desorbed material from the Si substrate, and moreover prevent contamination of the sample surface during cooling down. We found from other experiments that most of the usual contaminations of a cooled substrate surface can be considerably reduced by this procedure [59].

After reaching low temperature $(14.2 \mathrm{~K})$, the cell was kept for several hours under these conditions. Then the cleaning laser was switched off, and after establishing thermal equilibrium 


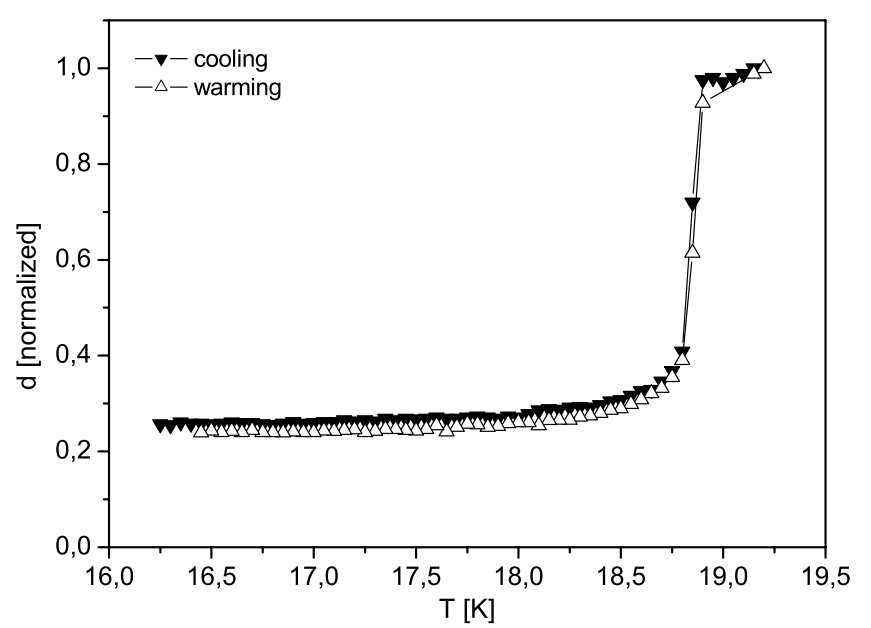

Figure 12. Depicted is the wetting behaviour of $\mathrm{D}_{2}$ on $\mathrm{Si}$. The thickness is normalized to the one in saturation above $T_{3}$.

between cell and substrate, an adsorption isotherm of $\mathrm{H}_{2}$ was performed. In the next step the cell was slowly cooled and warmed between 9 and $14.2 \mathrm{~K}$. The observed $T_{3}$ wetting behaviour and solid $\mathrm{H}_{2}$ film thickness determined, however, were about the same as measured for uncleaned Si substrates (figure 10). This shows that standard contaminations of the Si surface have no crucial influence on the wetting behaviour.

In the next step, after the second cooling, the temperature was fixed at $9 \mathrm{~K}$, and the laser annealing procedure, using the ruby laser set-up shown in figure 4, was performed. Upon annealing the $\mathrm{Si}$ substrate, the $\mathrm{H}_{2}$ film thickness increased by about two monolayers. Then we varied the temperature between 3.5 and $14 \mathrm{~K}$. The overall thickness in this temperature range, where only the solid film is present, remained indeed somewhat larger. Yet the typical $T_{3}$ wetting behaviour was observed, i.e., incomplete wetting of the solid hydrogen film. At the end of the experiment the surface quality of the Si was analysed by SEM. No sign of laser-induced damage were seen on the area where the measurements were done.

Although we observed only a small increase in the thickness of the solid film this result is promising and in qualitative agreement with the calculations of Esztermann et al [43]. Clearly more experiments are needed to check the influence of surface roughness on the growth of a solid hydrogen film and to compare more quantitatively with the theory. For that it is not only necessary to manipulate the surface of the substrate, but also to characterize this surface on the atomic scale. Therefore we have built a new set-up which allows both measurements of the film thickness, manipulation of the surface of the substrate, and in situ characterization via a low temperature scanning tunnelling microscope [59].

\section{Summary and conclusions}

An early theory [5] predicts that the induced strain from the substrate underneath an adsorbed solid film, e.g. due to lattice mismatch between substrate and film, is responsible for incomplete wetting of this film. This effect of dewetting can be amplified by the roughness of the substrate, as predicted by a recent theory [43]. Motivated by these ideas we investigated the effect of $T_{3}$ wetting of molecular $\mathrm{H}_{2}$ isotopes and checked the influence of the roughness of the substrate. For that we used different substrates, namely thermally evaporated gold films on glass and on 
bare Si wafers. The latter provide a much smoother surface. In addition we tried to manipulate the surface of the Si (by laser cleaning and annealing) in situ in order to vary the roughness properties. As a third parameter we tuned the interaction strength between adsorbate and substrate by preplating with different noble gases. We also used $\mathrm{D}_{2}$ as preplating material and, in this context, investigated the mixing properties and the dewetting of the solid phase of this binary system (by the two hydrogen isotopes).

It turned out that for all variations that we have considered for the substrates the usual dewetting of the solid phase below $T_{3}$ was observed. However, small differences in the adsorbed solid film thickness could be detected. Comparing the hydrogen thickness on $\mathrm{Au}$ and $\mathrm{Si}$ showed at $T \ll T_{3}$ an increase from about three to five solid layers of hydrogen. Additional laser cleaning of the Si surface did not result in any difference; however, a further increase of about two layers of solid hydrogen was measured on an annealed Si surface. These changes were less than expected. Still, they are very promising for the goal of achieving complete wetting of solid hydrogen films, and they are, at least qualitatively, in agreement with the recent calculations [43]. Further investigations on modifying the substrate properties (i.e., decreasing the surface roughness) are on the way, together with simultaneous (i.e., in situ) characterization of the substrates used and modified on the necessary nanometre scale.

\section{Acknowledgment}

This work was supported by the DFG-Schwerpunkt 'Wetting and Structure Formation at Interfaces' under grant Le 315/20.

\section{References}

[1] Dash J G and Puff R D 1981 Phys. Rev. B 24295

[2] Pandit R, Schick M and Wortis M 1982 Phys. Rev. B 265112

[3] Pandit R and Fisher M E 1983 Phys. Rev. Lett. 511772

[4] Huse D A 1984 Phys. Rev. B 296985

[5] Gittes T F and Schick M 1984 Phys. Rev. B 30209

[6] Dietrich S 1988 Phase Transitions and Critical Phenomena vol 12, ed C Domb and J L Lebowitz (London: Academic) p 1

[7] Schick M 1990 Liquids at Interfaces (Les Houches Session XLVIII) ed J Charvolin, J F Joanny and J Zinn-Justin (Amsterdam: Elsevier) p 415

[8] Youn H S and Hess G B 1990 Phys. Rev. Lett. 64443

[9] Youn H S, Meng X F and Hess G B 1993 Phys. Rev. B 4814556

[10] Hess G B 1991 Phase Transitions in Surface Films vol 2, ed H Taub, G Torzo, H J Lauter and S C Fain (New York: Plenum) p 357

[11] Migone A D, Dash J G, Schick M and Vilches O E 1986 Phys. Rev. B 346322

[12] Rieutord F, Simon R, Conradt R and Buschbaum P M 1997 Europhys. Lett. 37565

[13] Mistura G, Ancilotto F, Bruschi L and Toigo F 1999 Phys. Rev. Lett. 82795

[14] Krim J, Dash J G and Suzanne J 1984 Phys. Rev. Lett. 52640

[15] Bruschi L and Mistura G 2000 Phys. Rev. B 614941

[16] Daunt J G, Hedge S G, Tsui S P and Lerner E 1981 J. Low Temp. Phys. 44207

[17] Wu H and Hess G B 1998 Phys. Rev. B 576720

[18] Migone A D, Hofmann A, Dash J G and Vilches O E 1988 Phys. Rev. B 375440

[19] Leiderer P and Albrecht U 1992 J. Low Temp. Phys. 89229

[20] Albrecht U, Conradt R, Herminghaus S and Leiderer P 1996 Low Temp. Phys. 22117

[21] Klier J, Weichhard C and Leiderer P 2000 Physica B 284391

[22] Sohaili M, Klier J and Leiderer P 2001 J. Low Temp. Phys. 122249

[23] Tibus S, Sohaili M, Klier J and Leiderer P 2003 Low Temp. Phys. 29970

[24] Albrecht U, Evers P and Leiderer P 1993 Surf. Sci. 283419

[25] Albrecht U, Leiderer P and Kono K 1993 Surf. Sci. 283423 
[26] Fleischmann L, Bonn J, Degen B, Przyrembel M, Otten E W, Weinheimr C and Leiderer P 2000 J. Low Temp. Phys. 119615

[27] Fleischmann L, Bonn J, Bornschein B, Leiderer P, Otten E W, Przyrembel M and Weinheimr C 2000 Eur. Phys. J. B 16521

[28] Angrik J, Sohaili M, Klier J and Leiderer P 2003 Physica B 329435

[29] Klistner T and Pohl R O 1986 Phys. Rev. B 346045

[30] Kono K, Albrecht U and Leiderer P 1991 J. Low Temp. Phys. 83423

[31] Kono K, Albrecht U and Leiderer P 1991 J. Low Temp. Phys. 82279

[32] Monarkha Y P, Albrecht U, Kono K and Leiderer P 1993 Phys. Rev. B 4713812

[33] Otten O W 1994 Prog. Part. Nucl. Phys. 32153

[34] Backe H, Barth H, Balzhauser M, Bonn J, Degen B, Fleischmann L, Leiderer P, Moore R J, Otten E W, Schneller S, Przyrembel M and Weinheimer C 1994 Prog. Part. Nucl. Phys. 32173

[35] Otten O W 1995 Prog. Part. Nucl. Phys. 3826

[36] Davenport P H 1983 Prospects for Fusion ed W Marshal (Oxford: Clarendon)

[37] Craxton R S, McGroy R L and Sourer J M 1986 Sci. Am. 25560

[38] Wagner M and Ceperley D M 1994 J. Low Temp. Phys. 94161

[39] Wagner M and Ceperley D M 1996 J. Low Temp. Phys. 102275

[40] Vidali G, Ihm G, Kim H Y and Cole M W 1991 Surf. Sci. Rep. 12135

[41] Aziz R A 1984 Inert Gases ed M L Klein (Berlin: Springer) p 5

[42] Cheng E, Cole M C, Saam W F and Treiner J 1993 Phys. Rev. B 4818214

[43] Esztermann A, Heni M, Löwen H, Klier J, Sohaili M and Leiderer P 2002 Phys. Rev. Lett. 8855702

[44] Raether H 1986 Surface Plasmons (Berlin: Springer)

[45] Herminghaus S and Leiderer P 1989 Appl. Phys. Lett. 5499

[46] Azzam R M A and Bashara N M 1987 Ellipsometry and Polarized Light (Amsterdam: North-Holland)

[47] McCrakin F L, Passaglia E, Stromberg R R and Steinberg H L 1963 J. Res. Natl Bur. Stand. A 67363

[48] Archer R J 1963 Ellipsometry in the Measurement of Surface and Thin Films ed E Passaglia, R R Stromberg and J Kruger (Washington, DC: Symposium Proceedings) p 255

[49] Jellison G E, Lowndes D H, Mashburn D N and Wood R F 1986 Phys. Rev. B 342407

[50] Boneberg J 1993 Dynamische verfestigung von halbleiterschichten nach ns-laser-annealing PhD Thesis University of Konstanz, Konstanz

[51] Halsey G D 1948 J. Chem. Phys. 16931

[52] Frenkel J 1949 Kinetic Theory of Liquids (London: Oxford University Press)

[53] Hill T L 1949 J. Chem. Phys. 17520

[54] Vorberg J, Herminghaus S and Mecke K 2001 Phys. Rev. Lett. 87196105

[55] Mecke K R and Krim J 1996 Phys. Rev. B 532073

[56] Vidali G and Cole M W 1981 Surf. Sci. 11010

[57] Rauber S, Klein J R, Cole M W and Bruch L W 1982 Surf. Sci. 123173

[58] Schwartz C and Roy R J L 1986 Surf. Sci. Lett. 166141

[59] Zech M, Fubel A, Leiderer P and Klier J 2004 J. Low Temp. Phys. 137179 